\title{
AN EXAMPLE OF NON-HOMEOMORPHIC CONJUGATE VARIETIES
}

\author{
C. S. RAJAN
}

\begin{abstract}
We give examples of smooth projective varieties over complex numbers, in the context of connected Shimura varieties, which are not homeomorphic to a conjugate of itself by an automorphism of the complex numbers.
\end{abstract}

\section{Introduction}

Let $X$ be a quasi-projective variety defined over $\mathbb{C}$. Suppose $\sigma$ is an automorphism of $\mathbb{C}$. Denote by $X^{\sigma}:=X \times_{\sigma} \mathbb{C}$, the conjugate of $X$ by the automorphism $\sigma$ of $\mathbb{C}$, obtained by applying the automorphism $\sigma$ to the coefficients of the polynomials defining $X$. It is known that the varieties $X$ and $X^{\sigma}$ have the same Betti numbers. In [10], Serre gave an example where the topological spaces $X(\mathbb{C})$ and $X^{\sigma}(\mathbb{C})$ are not homeomorphic.

Recently, Milne and Suh [6] gave further examples in the context of connected Shimura varieties. Their method is to find a conjugate such that the reductive group underlying the Shimura datum is different, and then apply the super-rigidity results of Margulis.

Our examples are in the same context as that of Milne and Suh, but we work with Shimura's construction of canonical models [12]. Shimura's construction allows us to identify the adelic congruence subgroup defining the conjugate variety as a conjugate by an element of the adjoint group. We then appeal to Mostow rigidity and the failure of strong approximation (or non-triviality of class number) for the adjoint group to get at the desired examples. In our example, the congruent lattices defining the variety and its conjugate are commensurable. Earlier in [9], we observed using Shimura's construction coupled with the theorems of Labesse and Langlands on the mulitplicity of cusp forms for $S L(1, D)$, that a Galois twist of these spaces attached to $S L(1, D)$ over the reflex field preserves the spectrum of the Laplacian; this provides examples of locally symmetric spaces attached to a quaternion division algebra over a number field, which are isospectral but not isometric.

Apart from the work of Milne and Suh, many other constructions of nonhomeomorphic conjugate varieties have been constructed by different methods. We refer to $[1,2,4,5,11]$ and references cited in these papers for different facets of this problem. For example, in $[2,5]$, the construction of such examples is motivated by the problem of knowing whether the Galois group acts faithfully on the components of the moduli space of surfaces.

Received by the editors October 27, 2010. Revision received April 20, 2011.

1991 Mathematics Subject Classification. Primary 58G25; Secondary 22E55, 12A70. 


\section{The example}

Let $F$ be a totally real number field of degree at least two over $\mathbb{Q}$. Let $D$ be an indefinite quaternion division algebra defined over $F$. We assume that $D$ is split at exactly one real place, say $\tau_{1}$ of $F$. This assumption allows us to assume that the reflex field of $\left(F, \tau_{1}\right)$ to be $F$ itself. Let $V$ be a vector space of rank $n \geq 2$ over $D$, equipped with a hermitian inner product with respect to the standard involution on $D$. We assume that the inner product is definite on the spaces $V \otimes_{\tau} \mathbb{R}$, for all real embeddings $\tau$ of $F$ different from $\tau_{1}$. In particular, since we have assumed that the degree of $F$ is at least two, the form $h$ is anisotropic. Let $G$ be the group of unitary similitudes of $h$. We consider $G$ as an algebraic group defined over $F$, and let $G_{d}$ be the derived group of $G$. Denote by $P G$ the adjoint group attached to $G$, the group obtained by taking the quotient of $G$ modulo its centre. For an algebraic group $H$ defined over a number field $F$, we let $H_{\infty}=H(F \otimes \mathbb{R})$ be the real Lie group consisting of the real points of $H$. Under our assumptions, it follows that

$$
G_{d, \infty} \simeq S p(2 n, \mathbb{R}) \times \text { a compact group, } \quad n \geq 2 .
$$

Let $K_{\infty}$ be a maximal compact subgroup of $G_{d, \infty}$ and let $X=G_{d, \infty} / K_{\infty}$ be the non-compact symmetric space associated to $G_{d}$. By our assumptions, $X$ is isomorphic to the Siegel upper half-space $\mathbb{H}_{n}$ of dimension $n$. We also have a natural action of $G_{\infty}$ on $X$. Denote by $\mathbf{A}$ the adele ring of $F$, and by $\mathbf{A}_{f}$ the subring of finite adeles. Let $K$ be a compact open subgroup of $G\left(\mathbf{A}_{f}\right)$, and let $K_{d}=K \cap G_{d}\left(\mathbf{A}_{f}\right)$. Denote by

$$
\Gamma_{K}=G_{\infty} K \cap G(F) \quad \text { and } \quad \Gamma_{d, K}=G_{d, \infty} K_{d} \cap G_{d}(F),
$$

the corresponding arithmetic lattices in $G(F \otimes \mathbb{R})$ and $G_{d}(F \otimes \mathbb{R})$, respectively. We assume that $K$ is such that $\Gamma_{d, K}$ is torsion-free, and the natural inclusion $\Gamma_{d, K} \subset \Gamma_{K}$ is an isomorphism modulo the centre of $\Gamma_{K}$. By a congruence subgroup we will either mean a compact open subgroup contained in the group of finite adele points of the algebraic group, or the corresponding arithmetic lattice contained in the real points of the algebraic group.

By a theorem of Baily-Borel, the quotient space $X_{K}=\Gamma_{K} \backslash X$ is a connected, smooth, projective variety. The fundamental group $\bar{\Gamma}_{K}$ of the variety $X_{K}$ can be identified with the projection of $\Gamma_{K}$ to $P G_{\infty}$, and also with the lattice $\Gamma_{d, K}$ contained in $G_{d, \infty}$.

For an element $x \in G\left(\mathbf{A}_{f}\right)$, denote by $K^{x}$ the conjugate lattice $x^{-1} K x$, and by $\bar{x}$ its image in $P G\left(\mathbf{A}_{f}\right)$ (a bar on top will indicate the image in the adjoint group $P G$ ). Further, let $N\left(\overline{K_{d}}\right)$ denote the normalizer of $\overline{K_{d}}$ in $P G\left(\mathbf{A}_{f}\right)$, where $\overline{K_{d}}$ is the image of $K_{d}$ in $P G\left(\mathbf{A}_{f}\right)$. The desired example is provided by the following theorem.

Theorem 2.1. With notation and assumptions as above, suppose $\bar{x}$ does not belong to the set $N\left(\overline{K_{d}}\right) P G(F)$. Then $X_{K}$ and $X_{K^{x}}$ are conjugate by an automorphism $\sigma$ of $\mathbb{C}$, but the respective fundamental groups $\bar{\Gamma}_{K}$ and $\bar{\Gamma}_{K^{x}}$ are not isomorphic. In particular, $X_{K}$ and $X_{K^{x}}$ are not homeomorphic.

Proof. We first show that the varieties $X_{K}$ and $X_{K^{x}}$ are conjugate by an automorphism of $\mathbb{C}$. For this, we recall Shimura's theory of canonical models [12]. Let $\nu: G \rightarrow \mathbf{G}_{m}$ be the reduced norm. By class field theory, the subgroup $F^{*} \nu(K)$ of the 
idele group $\mathbf{A}^{*}$ defines an abelian extension $F_{K}$ of $F$. The reciprocity morphism of class field theory,

$$
\operatorname{rec}: \mathbf{A}^{*} / F^{*} \rightarrow \operatorname{Gal}\left(F^{a b} / F\right)
$$

defines an element $\sigma(x) \in \operatorname{Gal}\left(F^{a b} / F\right)$ by the prescription

$$
\sigma(x)=\operatorname{rec}\left(\nu(x)^{-1}\right) .
$$

As a consequence of the main theorem of canonical models in [12, Theorem 2.5, p. 159, Section 2.6], the variety $X_{K}$ has a model defined over the field $F_{K}$, and

$$
X_{K}^{\sigma(x)} \simeq X_{K^{x}}
$$

Thus, the varieties $X_{K}$ and $X_{K^{x}}$ are conjugate.

Suppose on the contrary, that $X_{K}$ and $X_{K^{x}}$ have isomorphic fundamental groups. Since these spaces are Eilenberg-Maclane spaces, there exists a homotopy equivalence

$$
\phi: X_{K} \rightarrow X_{K^{x}}
$$

Since the lattices are irreducible in $P G_{\infty}$ and the real rank of $P G$ is at least two, by Mostow rigidity [7], the spaces $X_{K}$ and $X_{K^{x}}$ are isometric.

Hence, there exists $\bar{g} \in P G_{\infty}$ such that

$$
\bar{g}^{-1} \bar{\Gamma}_{K^{x}} \bar{g}=\bar{\Gamma}_{K}
$$

Since the lattices $\bar{\Gamma}_{K}$ and $\bar{\Gamma}_{K^{x}}$ are arithmetic and commensurable, it follows by a theorem of Borel [3], that $\bar{g} \in P G(F)$. Hence, there is an element $g \in G(F)$ satisfying,

$$
g^{-1} \Gamma_{d, K^{x}} g=\Gamma_{d, K} .
$$

Consider now $G_{d}(F)$ embedded diagonally in $G_{d}\left(\mathbf{A}_{f}\right)$. By the strong approximation theorem for $G_{d}$, the closure of $\Gamma_{d, K}$ in $G_{d}\left(\mathbf{A}_{f}\right)$ can be identified with $K_{d}$. Further, the closure of $\Gamma_{d, K^{x}}$ in $G_{d}\left(\mathbf{A}_{f}\right)$ can be identified with $g^{-1} K_{d}^{x} g$, where we now consider $g \in G(F)$ as diagonally embedded in $G\left(\mathbf{A}_{f}\right)$. Hence, we have

$$
g^{-1} K_{d}^{x} g=g^{-1} x^{-1} K_{d} x g=K_{d} .
$$

Projecting to $P G$, we obtain

$$
\bar{g}^{-1} \bar{x}^{-1} \overline{K_{d}} \bar{x} \bar{g}=\overline{K_{d}}
$$

where $\overline{K_{d}}$ denotes the image of $K_{d}$ in $P G\left(\mathbf{A}_{f}\right)$. This implies that $\bar{x} \in N\left(\overline{K_{d}}\right) P G(F)$, contradicting our choice of $\bar{x}$.

2.1. Congruence subgroups with small normalizers. One way of producing congruence lattices $K$ and an element $x \in G\left(\mathbf{A}_{f}\right)$ satisfying the hypothesis of the Theorem, is to impose an additional arithmetical condition on the field $F$. Let $S$ be a finite set of places of $F$ containing the archimedean places and the finite places of $F$ at which $D$ is ramified. Let $S_{f}$ denote the subset of $S$ which are non-Archimedean, and $S^{\prime}$ the complement of $S$ in the collection of places of $F$.

We assume that the group $C_{F, S} / C_{F, S}^{2 n}$ is non-trivial, where $C_{F, S}$ is the $S$-class group of $F$ obtained by considering ideals without any $S$-component. Here $C_{F, S}^{2 n}$ is the subgroup consisting of the $2 n$-multiples of elements in $C_{F, S}$. In particular, for example, if $S$-class number of $F$ is divisible by 2 , then the above condition holds. 
Since we are working with groups of type $C_{n}$, for $v$ not in $S$, the group $G_{v}:=$ $G \times$ Spec $F$ Spec $F_{v}$ is split. We can assume that the groups $G_{d}, P G$ extend to Chevalley group schemes over the local ring $\mathcal{O}_{v}$ for $v \in S^{\prime}$. Thus, we have an exact sequence over $\mathcal{O}_{v}, v \in S^{\prime}$ of group schemes,

$$
1 \rightarrow \mathbf{G}_{m} \rightarrow G \rightarrow P G \rightarrow 1 .
$$

By Hilbert Theorem 90 for étale cohomology, we have a surjection $G\left(\mathcal{O}_{v}\right) \rightarrow$ $P G\left(\mathcal{O}_{v}\right)$ for $v \in S^{\prime}$. Further, from Bruhat-Tits theory, we know that the groups $P G\left(\mathcal{O}_{v}\right)$ are maximal compact and also maximal subgroups of $P G\left(F_{v}\right)$. We can also assume that the adele groups associated to $G$ and $P G$ are formed with respect to these classes of compact open subgroups.

Now let $K_{m}^{S}=\prod_{v \in S^{\prime}} G\left(\mathcal{O}_{v}\right)$ be a maximal compact subgroup of the group of $S$ adeles $G\left(\mathbf{A}^{S}\right)$, the subgroup of the adele group $G(\mathbf{A})$ having no $S$-component. Here by $\mathbf{A}^{S}$, we mean the subgroup of adeles of $F$ having no $S$-component. We choose a compact open subgroup of the form $K=K_{S} K^{S}$ satisfying the following:

- The group $K_{S}$ is a compact open subgroup in $G_{S_{f}}:=\prod_{v \in S_{f}} G\left(F_{v}\right)$.

- The group $K^{S}$ is a compact open subgroup of $K_{m}^{S}$.

- The subgroup $K_{d}^{S}:=K^{S} \cap G_{d}\left(\mathbf{A}^{S}\right)$ is normal in $K_{m}^{S}$.

- The arithmetic lattice $\Gamma_{d, K}$ is torsion-free.

This can be achieved by considering principal congruence subgroups at a finite collection of places not in $S$ of $F$.

Since $\overline{K_{m}^{S}}=\prod_{v \in S^{\prime}} P G\left(\mathcal{O}_{v}\right)$ is maximal in $P G\left(\mathbf{A}^{S}\right)$, it follows from the maximality of $P G\left(\mathcal{O}_{v}\right)$ and the fact that the groups $P G\left(F_{v}\right)$ are simple, that the normalizer $N\left(\overline{K_{d}^{S}}\right)$ of $\overline{K_{d}}$ in $P G\left(\mathbf{A}^{S}\right)$ is precisely $\overline{K_{m}^{S}}$.

Thus, to produce an element $x \in G\left(\mathbf{A}_{f}\right)$ such that $\bar{x}$ does not belong to the double coset $N\left(\overline{K_{d}}\right) P G(F)$, it is enough to show that $x$ does not belong to the set $Z\left(\mathbf{A}_{f}\right) G_{S_{f}} K_{m}^{S} G(F)$. For this, it is enough to work with the $S$-adele component $x^{S} \in G\left(\mathbf{A}^{S}\right)$ of $x$.

The reduced norm map $\nu: G \rightarrow \mathbf{G}_{m}$ induces a surjection $\nu: G\left(\mathbf{A}_{f}\right) \rightarrow \mathbf{A}_{f}^{*}$. Hence, we need to show that the image group

$$
\nu\left(Z\left(\mathbf{A}_{f}\right) G_{S_{f}} K_{m}^{S} G(F) \cap G\left(\mathbf{A}^{S}\right)\right)
$$

is a proper subgroup of $\left(\mathbf{A}^{S}\right)^{*}$. Since the image lands in the subgroup $\left(\mathbf{A}^{S}\right)^{* 2 n} \nu\left(K_{m}^{S}\right)$ $F^{*}$, we need to know that the group

$$
\left(\mathbf{A}^{S}\right)^{*} /\left(\mathbf{A}^{S}\right)^{* 2 n} F^{*} \nu\left(K_{m}^{S}\right)
$$

is non-trivial. Its image in the $S$-class group $C_{F, S}$ of $F$ lies in the subgroup $C_{F, S}^{2 n}$. Hence, if $C_{F, S} / C_{F, S}^{2 n}$ is non-trivial, we can find an element $x \in G\left(\mathbf{A}_{f}\right)$ such that $\bar{x}$ does not lie in the coset $N\left(\overline{K_{d}}\right) G(F)$.

Remark. The above construction is similar to and can be compared with the construction given by Vignéras in [13], where she works with fields with class numbers divisible by 2 .

Remark. It is of interest to produce examples of congruence subgroups $K$ such that $N\left(\overline{K_{d}}\right) P G(F)$ is not equal to $P G\left(\mathbf{A}_{f}\right)$ over an arbitrary field $F$ without imposing any arithmetical restrictions on $F$. This certainly seems plausible. For instance, if we 
are working with an arithmetical Riemann surface associated to an adelic congruence subgroup $K \subset S L_{1}(D)\left(\mathbf{A}_{f}\right)$, where $D$ is a quaternion division algebra defined over $F$, then $N(\bar{K}) / \bar{K}$ gives raise to automorphisms of the Riemann surface. As $K$ varies, generically one expects that this automorphism group is small. However, it seems to be quite delicate to produce such examples.

Let $S$ be a sufficiently large set of places of $F$ containing the archimedean places and also the places at which the group $G_{d}\left(F_{v}\right)$ is compact. The failure of strong approximation for the adjoint group $P G$ [8, Proposition 7.13] implies that the set $P G_{S} P G(F)$ is not dense in $P G(\mathbf{A})$, where $P G_{S}=\prod_{v \in S} P G\left(\mathbb{F}_{v}\right)$. Thus, there exists a sufficiently small compact open subgroup $M \subset P G\left(\mathbf{A}^{S}\right)$ such that $M P G_{S} P G(F)$ is not equal to the full adele group $P G(\mathbf{A})$, where $P G\left(\mathbf{A}^{S}\right)$ is the adele group associated to $P G$ without a $S$-component.

The problem can thus be reduced to the following question: given a nonArchimedean local field $F$, a reductive, non-anisotropic group $G$ defined over $F$, and a congruence subgroup $L \subset G(F)$, to show that there exists a congruence subgroup $K$ such that its normalizer in $G(F)$ is contained in $L$. D. Prasad has shown me how to construct such candidates of congruence subgroups with small normalizers: the details, however, are not only somewhat complicated but also have to be worked out.

\section{Acknowledgment}

My sincere thanks to Dipendra Prasad for many useful discussions, especially in the context of strong approximation for algebraic groups; to the referee for raising the question of making explicit the construction of the required congruence subgroups, which turns out to be an interesting question in itself; to A. R. Shastri and Ravi Vakil for pointing out references to recent literature.

\section{References}

[1] H. Abelson, Topologically distinct conjugate varieties with finite fundamental group, Topology 13 (1974), 161-176.

[2] E. Artal Bartolo, J. Carmona Ruber and J. I. Cogolludo Agustín, Effective invariants of braid monodromy, Trans. Amer. Math. Soc. 359(1) (2007), 165-183 (electronic).

[3] A. Borel, Density and maximality of arithmetic subgroups, J. Reine Angew. Math. 224 (1966), 78-89.

[4] F. Charles, Conjugate varieties with distinct real cohomology algebras, J. Reine Angew. Math. 630 (2009), 125-139.

[5] R. W. Easton and R. Vakil, Absolute Galois acts faithfully on the components of the moduli space of surfaces: a Belyi-type theorem in higher dimension, Int. Math. Res. Not. IMRN (2007), no. 20, Art. ID rnm080, 10pp.

[6] J. S. Milne and J. Suh, Nonhomeomorphic conjugates of connected Shimura varieties, Amer. J. Math. 132(3) (2010), 731-750.

[7] G. D. Mostow, Strong rigidity of locally symmetric spaces, Princeton University Press, Princeton, NJ, 1973. Annals of Mathematics Studies, No. 78.

[8] V. Platonov and A. Rapinchuk, Algebraic groups and number theory, Vol. 139 of Pure and Applied Mathematics, Academic Press Inc., Boston, MA, 1994. Translated from the 1991 Russian original by Rachel Rowen.

[9] C. S. Rajan, On isospectral arithmetical spaces, Amer. J. Math. 129(3) (2007), 791-806.

[10] J.-P. Serre, Exemples de variétés projectives conjuguées non homéomorphes, C. R. Acad. Sci. Paris 258 (1964), 4194-4196. 
[11] I. Shimada, Non-homeomorphic conjugate complex varieties, in 'Singularities-Niigata-Toyama 2007', 56, Adv. Stud. Pure Math., Math. Soc. Japan, Tokyo, 2009, 285-301.

[12] G. Shimura, On canonical models of arithmetic quotients of bounded symmetric domains, Ann. Math. 91(2) (1970), 144-222.

[13] M.-F. Vignéras, Variétés riemanniennes isospectrales et non isométriques, Ann. Math. (2) 112(1) (1980), 21-32.

Tata Institute of Fundamental Research, Homi Bhabha Road, Bombay - 400 005, India E-mail address: rajan@math.tifr.res.in 